INVESTIGACIÓN/RESEARCH

Recibido: 13/10/2013-----Aceptado: 20/12/2013-----Publicado: 15/03/2014

\title{
LAS REDES SOCIALES EN LAS UNIVERSIDADES ESPAÑOLAS
}

Santiago Alonso García ${ }^{1}$. Universidad de Castilla La Mancha. España. santiago.agarcia@uclm.es

María del Mar Alonso García. Universidad de Málaga. España.

mariadelmaralonsogarcia@gmail.com

\section{RESUMEN}

Las Universidades españolas en pleno proceso de adaptación al Espacio Europeo de Educación Superior, han incorporado a sus canales de comunicación las redes sociales, como un canal de comunicación directo y rápido con el alumnado. La web 2.0 es un catalizador en el proceso de aprendizaje en el que se integran los objetivos del EEES y las necesidades de la sociedad actual de comunicarse, con las personas y las instituciones de forma asincrónica. En esta investigación se analiza los perfiles de redes sociales (Facebook y Twitter) que tienen algunas de las mejores Universidades de España. De esta información se puede extraer la implicación de las instituciones académicas en sus publicaciones diarias, el tipo de contenidos o la interactuación con sus seguidores o fans.

PALABRAS CLAVE: Redes sociales - Comunicación - Universidad - Facebook Twitter - Educación - Internet

\footnotetext{
${ }^{1}$ Santiago Alonso García: Facultad de Ciencias de la Educación (Campus Albacete) de la Universidad de Castilla La Mancha. España.

Correo: santiago.agarcia@uclm.es
} 


\title{
SOCIAL MEDIA IN SPANISH UNIVERSITIES
}

\begin{abstract}
Spanish universities in the process of adaptation to the European Higher Education have joined their communication channels social media, as a direct communication channel with students. Web 2.0 is a catalyst in the process of learning that integrate the objectives of the EEES and needs current society, with people and institutions asynchronously. This research analyzes the social networking profiles (Facebook and Twitter) that have some of the best universities in Spain. From this information we can extract the involvement of academic institutions in their daily publications, type of content or interacting with their followers or fans.
\end{abstract}

KEYWORDS: Social media - Communication - University - Facebook - Twitter Education - Internet

\section{INTRODUCCIÓN}

\subsection{Las TT.II.CC. en el Espacio Europeo de Educación Superior}

Actualmente el fenómeno de la globalización y la continua aparición y desarrollo de avances científicos y tecnológicos están haciendo que la Universidad como parte de la sociedad se encuentre inmersa en una época de cambios. La función formativa e investigadora de la Universidad se ve afectada en el actual contexto socio-económico por lo que ha sido denominada la sociedad de la información y el conocimiento. En este contexto trataremos de abordar el proceso de cambio y adaptación de las instituciones educativas: universidades, en el Espacio Europeo de Educación Superior. Este proceso se dirige hacia una Universidad renovada en la que juegan un importante papel las TIC y las redes sociales en el proceso de transformación y adaptación, en el cual el alumno y el docente son dos de los actores principales.

Los retos que se han planteado en la Universidad tienen una triple vertiente formativa:

- Labor formativa para la investigación

- Tarea de capacitación para preparar a profesionales

- Formación de ciudadanos que promuevan el desarrollo personal en la sociedad

La formación en cada uno de estos aspectos debe ser elaborada, estructurada y desarrollada en todos los niveles educativos, aunque en algunos se debe hacer más hincapié que en otros. La Educación Superior en Europa ha afrontado un periodo de reformas en sus principios para actualizar y mejorar su labor formativa, promoviendo la excelencia en cada uno de los ámbitos en los que está presente. Con este proceso de convergencia se persigue obtener una apertura y movilidad entre los estudiantes de diferentes instituciones para permitir una mejora en la competitividad. 
La realidad actual demanda una respuesta adaptativa a las nuevas necesidades de formación en una sociedad multicultural y ávida de conocimiento. Desde el punto de vista económico, (Aguilera, 2000) ya señalaba como realidades a tener en cuenta el desempeño y la movilidad profesional que necesitan de la formación en la Universidad el conocimiento de nuevas habilidades (informática, idiomas etc.) y preparación especializada para estas nuevas circunstancias. De igual forma la formación debe ser más polivalente y adaptada a los perfiles de trabajo que los futuros profesionales tendrán que cubrir. Por otro lado, nos enfrentamos a una etapa marcada por la gran aparición de conocimientos y actualización de los mismos. Debido a esto el reciclaje formativo ha de ser continuo para poder enfrentarse a la realidad profesional del día a día. En este escenario la formación tiene que ser flexible y contener los recursos necesarios para favorecer el aprendizaje continuo a lo largo de la vida.

Entre otras las funciones que tiene actualmente la Universidad son las siguientes (Marqués, 2001):

- Formar a personas cultas con capacidades analíticas, críticas, receptivas e independientes que usen las TIC y sean capaces de trabajar de forma cooperativa

- Contribuir al desarrollo socio-económico y al desarrollo del entorno en el que se ubica dicha institución universitaria

- Desarrollar investigaciones científicas (teóricas y aplicadas) en colaboración con otras empresas e instituciones

- Impartir los niveles superiores de enseñanza, ofreciendo una formación teórica y práctica, adaptada a las necesidades de la sociedad y la economía. Entre esta formación se incluye también la del profesorado y la de las personas en general que quieran enriquecer sus conocimientos, dentro del proceso de formación permanente.

- Ofrecer una perspectiva crítica, siendo ejemplo de actuación en el contexto ético- social.

Por otro lado Sangrá y Gonzalez Sanmamed (2004) y Tomás, Feixas y Marqués (1999) recogen las diferentes características que se exponen en las universidades y que tienen un impacto directo en la sociedad de la información:

- Transformación de la naturaleza del trabajo y de la organización de la producción.

- Aumento del profesorado y transformación de la función docente universitaria.

- Necesidad de una renovación continua de los conocimientos.

- Ampliación del número de estudiantes y diversificación de su perfil.

- Creciente acercamiento entre la Universidad y el sector empresarial.

- Nueva forma de gestión universitaria.

- Revolución científico-técnica que empieza a crear una nueva cultura.

- Intercambios con Europa cada vez más frecuentes e intensos.

- Realización de investigaciones cada vez más interdisciplinares y grupales. 
Ante estas circunstancias se hace cada vez más imprescindible una reorganización en el sistema universitario, que pueda abordar las características anteriormente mencionadas y las necesidades de innovación en las formas de generación y transmisión del conocimiento que se están produciendo en la sociedad actual.

En este contexto los países europeos han tratado de establecer un marco de actuación en el que se intente dar respuesta a todas las inquietudes planteadas. Una plataforma que no se quede en un espacio común sino que se traslade a las estructuras, las metodologías y una nueva reconceptualización de la docencia universitaria basada en un proceso de adaptación a las necesidades diversas y competitivas de la sociedad actual.

En este contexto de adaptaciones los elementos como la competitividad, la movilidad y la diversidad presentes y necesarios en el nuevo Espacio Europeo surge también la necesidad de la promoción de la búsqueda de la calidad y excelencia en todos los procesos formativos. De igual forma, es destacable el profundo cambio metodológico en el que el rol del docente y el estudiante cambian.

En definitiva, como dice Zabala (2006) se trata de volver a los principios generales de la docencia, basados en que el aprendizaje sea un proceso formativo que no esté orientado a la continúa acumulación de conocimientos, sino que por el contrario esté basado en el afianzamiento y desarrollo de competencias profesionales.

\subsection{Web 2.0: las redes sociales en la Universidad.}

La web 2.0 es un catalizador en el proceso de aprendizaje en el que se integran los objetivos del Espacio Europeo de Educación Superior y las necesidades de la sociedad actual de comunicarse, con las personas y las instituciones de forma asincrónica. En este sentido se convierten en una herramienta básica del aprendizaje activo y colaborativo.

Desde el inicio de las redes sociales (Facebook, Linkedin, Twitter, Bebo, entre otras) son millones los usuarios que han sucumbido a su funcionalidad y conexión (FoonHew, 2011). Las redes sociales permiten un nuevo espacio de comunicarse en tiempo y forma, de relacionarse y de crear comunidades (Varas Rojas, 2009).

Como señala Castells (2001), la sociedad red, es una sociedad cuya estructura social está construida en torno a redes de información a partir de las tecnologías. Internet no es simplemente una tecnología, es el medio de comunicación que constituye una parte importante de la forma organizativa en nuestra sociedad. Mediante la Red, profesores y alumnos tienen acceso a material curricular, de formación de profesorado y otros materiales de aprendizaje.

Para comprender las posibilidades que aporta internet en el contexto educativo es necesario tener claro los conceptos de tecnología física (cable, software, velocidad de transmisión, etc.) herramientas (aplicaciones que permiten al usuario utilizar los 
servicios de la red) y entornos (virtuales o plataformas, cuya función principal es facilitar la distribución y difusión de información y el acceso a las herramientas para la comunicación entre profesores y alumnos).

Con el nuevo Espacio Europeo de Educación Superior el uso de las TT.II.CC. y la Web 2.0 se hace indispensable para situar al alumno en este nuevo escenario de aprendizaje. Al mencionar el concepto de Red Social, como dice el autor Celaya (2008:92) es "un lugar en internet donde las personas publican y comparten todo tipo de información personal y profesional con terceras personas conocidas y absolutos desconocidos".

Orihuela (2008), por otro lado, menciona las redes sociales como "nuevos espacios virtuales en los que nos relacionamos y en los que construimos nuestra identidad"; además según el autor, "también funcionan a modo de sistema de filtro y de alerta en la medida en que permiten un ajuste del flujo de información que recibimos en función de nuestros intereses y de los intereses de aquellos en quienes confiamos".

Es conveniente destacar que antes de que apareciera la Web 2.0 los principales soportes de contacto en internet eran el correo electrónico y otras herramientas como el foro o el chat. Sin embargo con la llegada de las redes sociales, los usuarios pueden conocer a otras personas de forma en línea y acceder a los contactos de amigos comunes, descubriendo a personas con intereses, objetivos y gustos afines (Orihuela, 2008).

En último lugar se debe hacer hincapié en las ventajas que aporta este tipo de aplicaciones para los usuarios como son: el dinamismo, la confluencia de personas con intereses comunes y la facilidad para la difusión de un determinado mensaje (García Orosa, 2009: 126).

\section{OBJETIVOS}

El principal objetivo de este artículo deriva de la necesaria implantación de las TT.II.CC. en el ámbito universitario por lo que la cercanía entre la Institución y el alumnado comienza a manifestarse desde el momento mismo en que las Universidades entran en el mundo virtual de las Redes sociales.

\section{METODOLOGÍA}

Para esta investigación se ha utilizado una metodología cualitativa; el análisis de contenido de los perfiles de Facebook y Twitter (las dos redes sociales de mayor uso por los internautas españoles) de las siete mejores Universidades de España según el Ránking Iberoamericano 2012: Universitat de Barcelona, Universitat Autónoma de Barcelona, Universidad Complutense de Madrid, Universitat de Valencia, Universitat Politécnica de Catalunya, Universidad Autónoma de Madrid y Universidad de Granada. 
Estas Universidades se encuentran entre los 10 principales de Facebook y Twitter de las Universidades Españolas, en referencia a sus publicaciones e interactuación con los alumnos y la sociedad. En las variables e ítems seleccionados, se ha contemplado el número de seguidores, la media diaria de publicaciones en una semana, el tipo de publicación, etc entre otras variables.

\section{RESULTADOS}

El uso de Facebook y Twitter en las Universidades. Como se puede observar en el análisis realizado los internautas utilizan Facebook y Twitter y están conectados con sus Universidades. Las Universidades con mayor número de seguidores/fans son las Universidades de Granada, Barcelona, Valencia y la Universidad Complutense de Madrid. Esto indica que sus alumnos eligen este canal de comunicación para estar conectados con la institución académica. La Universidad Politécnica de Catalunya es la Universidad con un menor número de seguidores en ambos canales.

En referencia a las publicaciones diarias realizadas, tras analizar la media semanal durante el mes de febrero, debemos incidir que hay variaciones de una Universidad a otra. Las Universidades con la media de post diarios más elevada son la Universidad de Valencia y la Universidad de Granada, en el perfil de Facebook con una media de cuatro publicaciones diarias. La Universidad Politécnica de Catalunya es la que registra la media más baja de publicaciones en el perfil de Facebook con un post diario. Sin embargo en la red social Twitter el número de publicaciones medias aumenta y las Universidades que registran una media más alta en orden descendiente son; la Universidad de Granada, la Universidad Autónoma de Madrid, la Universidad Politécnica de Catalunya y la Universidad de Barcelona.

La interactuación con los seguidores/fans se mide por los clicks que han recibido las publicaciones, las impresiones contabilizadas y los comentarios insertados. Esto constata la funcionalidad del canal de comunicación y la opinión del alumnado. La mayoría de las Universidades analizadas presentan un nivel alto de interactuación, que indica que sus publicaciones reciben feedback (me gusta, impresiones y comentarios) por parte del alumnado. La Universidad que registra una interactuación baja en el perfil de Twitter es la Universidad Complutense de Madrid, esto corresponde con la baja media de publicaciones diarios de tweets.

En los perfiles de social media, se publican noticias, videos o fotografías que muestran información de interés para el alumno, relacionada con su ámbito académico, social y cultural. Al observar los perfiles de las siete Universidades se puede observar que predominan unos tipos de publicaciones sobre otras. Todas las Universidades publican noticias con enlaces que redireccionan al alumno a la web de la Universidad, pero la Universidad de Valencia y la Universidad de Barcelona son las que realizan publicaciones alternativas de videos, noticias y fotografías. El resto de Universidades optan por las publicaciones de noticias y fotografías, excepto la 
Universidad de Complutense de Madrid y la Universidad Politécnica de Catalunya que solo publican noticias.

Este análisis nos aporta la adaptación de las Universidades a la web 2.0 y a las redes sociales. El tiempo de permanencia diario en las redes sociales ha aumentado y ellos comparten, crean y comentan contenidos, y en numerosas ocasiones están relacionados con el ámbito académico.

Los canales de social media cada vez ocupan un lugar más importante, la universidad, los docentes y los alumnos, deben estar preparados para comenzar a utilizar estos canales de comunicación en el ámbito educativo, porque tal como se puede extraer de los resultados las Universidades que tienen mayor número de seguidores poseen una mayor interactuación con sus alumnos y sus publicaciones tienen una mayor viralidad a través de los medios digitales.

\section{DISCUSIÓN}

De esta investigación podemos extraer las siguientes conclusiones:

- Siete de las mejores Universidades españolas se están adaptando a la web 2.0 y se están integradas en los perfiles más utilizados por los españoles (Facebook y Twitter). Como se ha podido extraer de esta investigación las Universidades españolas analizadas están integradas en la web 2.0 y han abierto los canales de comunicación de social media con sus públicos. Esto supone una adaptación importante al contexto actual y a la sociedad de la comunicación. A través de Facebook y Twitter la comunicación es directa, instantánea y asincrónica. En relación con el Espacio Europeo de Educación Superior esto contribuye a la formación de alumnos con capacidades analíticas, críticas, receptivas e independientes que usen las TIC y sean capaces de trabajar de forma cooperativa.

- Cuatro de las siete Universidades con un número de seguidores más elevado tienen una difusión mayor de sus publicaciones y sus seguidores comparten con su red de contactos la información en mayor medida. Esto favorece la difusión de la información y el aprendizaje colaborativo. Este canal de comunicación se convierte en un punto de referencia del que se puede obtener información de la institución académica y a través del cual se puede difundir información de interés académico y cultural, que contribuya a la formación de pensamientos críticos y a la interactuación.

- Las siete Universidades españolas interactúan con sus seguidores a través de los perfiles de Facebook y Twitter, sus seguidores no solo comparten información sino que también promocionan, consultan y recomiendan. Cuatro de ellas lo hacen en mayor medida. Se puede observar en los perfiles de los muros, cómo intervienen los usuarios, con consultas y opiniones. En estas expresiones se puede observar el tipo de lenguaje que usan para escribir, con caracteres abreviados y con un registro informal. Se percibe en tres de las siete universidades que mayor número de seguidores tienen, la interactuación y el volumen de consultas es mayor. También se puede constatar que el 
registro en estas Universidades tiende a ser menos informal y el estilo de la redacción de las consultas está más cuidado.

- Tan solo en dos de las mejores Universidades españolas se realiza una publicación proporcional en la semana de videos, noticias y fotografías. Coincide que cuatro de las universidades que realizan un menor número de publicaciones, el contenido suele ser noticias y enlaces, un contenido menos elaborado y más simple que las otras Universidades. Esto muestra que las Universidades que tienen mayor número de alumnos y favorecen la intervención, publican un contenido más elaborado y cuidado, que busca el interés y la interactuación del seguidor o fan. Esto se puede percibir por el texto y la redacción de los mensajes que se publican.

\section{REFERENCIAS}

Aguilera, J.M. (2000). El paradigma de la comunicación organizacional. Madrid. CECSA.

Almansa, A. Fonseca, O. y Castillo, A. (2013). Redes sociales y jóvenes. Uso de Facebook en la juventud colombiana y española. (DOI: http://dx.doi.org/10.3916/C40-2013-03-03).

De Haro, J.J. (2010). Redes sociales para la educación. Madrid. Anaya.

De la Torre, A. (2009). Nuevos perfiles en el alumnado: la creatividad en nativos digitales competentes y expertos rutinarios. Revista Universidad y Sociedad del Conocimiento.

Escamilla, A. (2009). Las competencias básicas. Claves y propuestas para su desarrollo en los centros. Barcelona. Graó

Espuny, C.; González y Gisbert. M. (2010). ¿Cuál es la competencia digital del alumnado al llegar a la Universidad?. Datos de una evaluación cero. Enseñanza \& Teaching, 28, 2, 113-137.

Flores, J.M. (2009). Nuevos modelos de comunicación, perfiles y tendencias en las redes sociales. Comunicar, 33: 70-80.

Gómez, M., Roses, S. y Farias, P. (2012) El uso académico de las redes sociales en universitarios. (DOI: http://dx.doi.org/10.3916/C38-2012-03-04).

Junco R. (2012). The relationship between frecuency of Facebook use, participation in Facebook activities, and student engagement. Computers and Education, 58, 1, 160-169. 
Ranking Iberoamericano 2012 de las mejores Universidades españolas. www.scimagoir.com/pdf/ranking_iberoamericano_2012.pdf 25/02/2013)

Sangrá, A. y González Sanmarned, M. (Coords). (2004). La transformación de las universidades a través de las TICS, discursos y prácticas. Barcelona. UOC.

Varas Rojas, L.E. (2009). Imaginarios sociales que van naciendo en comunidades virtuales: Facebook, crisis analógica, futuro digital. IV Congreso Online del Observatorio para la Cibersociedad, november 12-29 (www.cibersociedad.net/congres2009/actes/html/com_imaginarios-sociales-que-vannaciendo-en-comunidades-virtuales-facebook_709.html). Consultado: 28/02/2013.

\section{Santiago Alonso García}

Profesor en Colegio de Educación Infantil y Docente en la Facultad de Ciencias de la Educación de Albacete, ha colaborado en multitud de Congresos de Educación y ha participado en comunicaciones nacionales e internacionales.

\section{Ma del Mar Alonso García}

Técnica de Comunicación y Marketing en la Fundación General de la Universidad de Granada y Doctoranda de Dirección Estratégica de Comunicación. Ha trabajado con un grupo óptico en el desarrollo y la gestión de la comunicación corporativa y gestiona los perfiles en social media de la Fundación de la Universidad de Granada 